\title{
Reimmunization after bone marrow transplantation - current recommendations and perspectives
}

C.M. Machado ${ }^{1,2}$
Correspondence

C.M. Machado

Laboratório de Virologia, LIM-52

Av. Dr. Enéas C. Aguiar, 470

05403-000 São Paulo, SP

Brasil

Fax: +55-11-3063-2659

E-mail: clarimm@usp.bror

machado@aol.com

Presented at the I Symposium on Advances in Medical Research, Institute of Medical Investigation Laboratories, HC-FMUSP, São Paulo, SP, Brazil, March 21-22, 2003.

Publication supported by FAPESP.

Received June 17, 2003 Accepted September 9, 2003
${ }^{1}$ Laboratório de Virologia, LIM-52, Instituto de Medicina Tropical de São Paulo, and ${ }^{2}$ Divisão de Transplante de Medula Óssea, Disciplina de Hematologia, Faculdade de Medicina, Universidade de São Paulo, São Paulo, SP, Brasil

\begin{abstract}
Autologous and allogeneic bone marrow transplantation (BMT) recipients lose immune memory of exposure to infectious agents and vaccines accumulated through a lifetime and therefore need to be revaccinated. Diphtheria toxoid, tetanus toxoid, pertussis vaccine (children $<7$ years old), Haemophilus influenzae type b conjugate, 23valent pneumococcal polysaccharide, inactivated influenza vaccine, inactivated polio vaccine and live-attenuated measles-mumps-rubella vaccine are the currently recommended vaccines to be included in a vaccination program after BMT. For most of them, the best time to vaccinate, the number of vaccine doses and/or the duration of immunity after vaccination have not been established. Vaccination protocols vary greatly among BMT centers, suggesting that the lack of sufficient data has not permitted the formulation of reliable recommendations. The use of other vaccines and the perspectives for different vaccination protocols are analyzed in this review.
\end{abstract}

Key words

- Bone marrow

transplantation

- Vaccination

- Autologous

- Allogeneic

- Toxoid

- Polysaccharide

\section{Introduction}

The conditioning regimen used in marrow graft recipients ablates normal and abnormal immunohematopoietic elements and prepares the marrow microenvironment for the donor marrow to develop. The repopulation of the immune system is dependent on appropriate nesting, proliferation, maturation and differentiation of donor cells (1). Ultimately, the recipients lose immune memory of exposure to infectious agents and vaccines accumulated throughout their lives.

The loss of protective immunity to agents such as tetanus, poliovirus, and measles has been consistently demonstrated in patients submitted to allogeneic and autologous bone marrow transplantation (BMT), and consequently a reimmunization program is necessary to ensure immunity (2-5). Several surveys regarding reimmunization after BMT have demonstrated that vaccination protocols vary greatly among BMT centers, with insufficient data to establish solid recommendations (6-9).

The European Group for Blood and Marrow Transplantation, the Centers for Disease Control and Prevention, the Infectious Disease Society of America, and the American Society of Blood and Marrow Transplantation have recommended that the following vaccines be included in reimmunization pro- 
tocols for autologous, syngeneic and allogeneic BMT recipients: diphtheria toxoid, tetanus toxoid, pertussis vaccine (children $<7$ years old), Haemophilus influenzae type b (Hib) conjugate, 23-valent pneumococcal polysaccharide, inactivated influenza vaccine, inactivated polio vaccine and live-attenuated measles-mumps-rubella vaccine (10).

The present paper reviews the current recommendations and highlights the topics that deserve further investigation (Table 1).

\section{Vaccines currently recommended in reimmunization protocols}

\section{Diphtheria toxoid}

Immunity to diphtheria wanes over time. Lum et al. (11) showed that while $100 \%$ of the BMT patients with immune donors had antibodies to diphtheria within the first 100 days after transplantation, about $30 \%$ of them lost immunity thereafter. Increasing susceptibility (up to 40\%) was noticed in those with chronic graft-versus-host disease (GVHD). Other investigators evaluating long-term diphtheria immunity showed that only $54.5 \%$ of the patients still had antibodies to diphtheria with barely protective antitoxin levels one year after BMT (12).

There is evidence that multiple doses are more effective than a single dose in allogeneic recipients without chronic GVHD vac-

Table 1. Recommended immunizations after bone marrow transplantation (BMT).

\begin{tabular}{llcc}
\hline Vaccines & Time after BMT & No. of doses & Vaccine type \\
\hline Tetanus and diphtheria & After 4 months* & 3 doses & Toxoid \\
H. influenzae & After 4 months* & 2 doses & Conjugate polysaccharide \\
Polio & After 4 months* & 3 doses & Inactivated virus \\
Pneumococcal & After 12 months & 1 dose? & Polysaccharide \\
Hepatitis B & After 4 months* & 3 doses & Recombinant \\
Influenza & Yearly & 1 dose/year & Inactivated virus \\
MMR & After 2 years & 1 dose? & Attenuated virus
\end{tabular}

$\mathrm{MMR}=$ measles, mumps and rubella.

*Evidence recommends an early start. cinated 2 to 6 years after BMT (13).

Chronic GVHD seems to interfere with the response to vaccination 4 months after BMT (9). Although diphtheria vaccination starting one year after transplantation has been mostly recommended, no definitive data are available concerning the best time to start vaccination.

\section{Tetanus toxoid}

There is contrasting information concerning the persistence of tetanus immunity one year after BMT. Some investigators have observed sustained immunity in long-term allogeneic BMT survivors irrespective of toxoid administration pre- or post-transplantation (11). On the other hand, Ljungman et al. (3) observed that only $50 \%$ of the patients who were immune prior to transplantation sustained tetanus immunity for one year.

More recently, Parkkali et al. (14) also observed an increasing reduction in the mean concentration of anti-tetanus antibodies in this population. In the cited study, the patients were randomized to start early (6 months) or late (18 months) tetanus reimmunization. Before vaccination, $90 \%$ of the patients in the early group were immune to tetanus in contrast to $70 \%$ in the late group. Both schedules were equally immunogenic. However, in the late group, the recipient's antibody response after the first and second vaccine doses was correlated with donor's antibody levels, suggesting that donor immunity affects recipient response to tetanus toxoid.

Acute GVHD does not seem to interfere with the response to tetanus vaccination (14). Some studies on chronic GVHD did not show an effect on the response to vaccination (3), while others suggested that chronic GVHD may interfere with the intensity of the response (below four-fold rises) or with the duration of tetanus immunity after vaccination $(11,14)$. Although most BMT centers start tetanus immunization one year after 
transplantation, currently available information does not justify postponing tetanus immunization for more than 6 months after BMT (14).

The correlation of donor antibody levels with the response to tetanus vaccination indicates that prospective studies randomly vaccinating the donors before marrow harvesting should be conducted in this setting to better evaluate the need for donor vaccination in reimmunization programs.

\section{Pneumococcal polysaccharide}

BMT recipients are particularly at risk to develop life-threatening pneumococcal infections due to functional hyposplenism as a result of pre-transplant total body irradiation and chronic GVHD.

The currently available pneumococcal vaccines are the 14- and 23-valent polysaccharide vaccines and the 7 -valent conjugate vaccine. The polysaccharide vaccines contain only pure polysaccharides and require mature function of the immune system for a maximal response. The vaccines are therefore poorly immunogenic in transplant populations. Moreover, the polysaccharide vaccine does not cover $20 \%$ of the commonly pathogenic strains and immunized patients remain susceptible to them (9).

Evaluating a 14-valent pneumococcal vaccine in allogeneic BMT patients, Winston et al. (15) observed that pre- and postvaccination levels were significantly lower in BMT recipients compared to normal control subjects. Multiple regression analysis showed that vaccination within the early post-transplant period and corticosteroid therapy of GVHD were the two factors influencing the antibody response.

Other investigators have also observed decreasing pneumococcal antibody levels over the first year after BMT and a poor antibody response to a 23 -valent pneumococcal vaccine (16-19).

Little information is available regarding the impact of GVHD on the response to the pneumococcal vaccine since even nonGVHD patients are poor responders. Immunization of the donors before marrow harvesting did not influence the level of specific antibodies one year or more after transplantation $(16,20)$.

The data from most of the pneumococcal vaccine studies suggest that the key factor affecting response is possibly time to vaccination and consequently vaccination should be recommended after the second year of transplantation or even later $(15,21)$. However, impaired serum opsonic activity is expected during the first year after transplantation when life-threatening pneumococcal infections pose a greater risk. Thus, the currently available vaccine is not of substantial additional help in preventing pneumococcal infection during the first year post-transplant.

Long-term survivors without chronic GVHD are at lower risk for pneumococcal infection and probably only a few of them would benefit from vaccination. Among chronic GVHD patients, the use of corticosteroids affects their response to vaccination, rendering this strategy at least questionable.

The benefit of the new 7-valent pneumococcal conjugate vaccine has been recently investigated in allogeneic hematopoietic cell transplantation. Donors were randomized to receive or not one dose of the conjugate vaccine 7 to 10 days before transplantation. The recipients received three doses of the vaccine at 3,6 and 12 months after BMT. Protective immunity was achieved earlier in patients whose donors had been vaccinated, but after the three doses of the vaccine, protective immunity was similar in the two groups (22).

More studies are needed to better evaluate the effectiveness of the conjugate vaccine in BMT recipients. Due to the limited coverage of pneumococcal pathogenic strains by the 7-valent conjugate vaccine, prolonged 
prophylactic oral penicillin should be associated with the vaccination to prevent pneumococcal infection after BMT.

\section{Haemophilus influenzae type b conjugate}

Polysaccharide vaccines were not sufficiently immunogenic to the immature immune system of children and the preliminary results of Hib vaccination were disappointing. A new generation of polysaccharideprotein conjugated vaccines was developed and proved to be more immunogenic in children and also in BMT patients. The polysaccharide antigen is conjugated to a protein such as tetanus or diphtheria toxoid or both. Data from Hib vaccination studies in BMT patients have demonstrated that at least two doses of the conjugated vaccine are necessary to ensure protective antibody levels $(23,24)$. Comparing multiple Hib vaccination schedules after BMT, Vance et al. (24) observed that protective levels were achieved after the third or second dose of Hib vaccine in patients starting immunization at 3 or 6 months after BMT, respectively.

Parkkali et al. (18), using a single dose of diphtheria conjugated Hib vaccine in 45 BMT recipients randomized to start vaccination at 6 or 18 months, observed that both schedules were equally immunogenic. Since the greatest risk for infection by encapsulated bacteria occurs during the first 2 years after BMT, early-start schedules should be preferred in this setting.

Donor and recipient immunization with Hib vaccine before BMT proved to be more effective than recipient vaccination after BMT as demonstrated by a higher antibody concentration in patients as early as 3 months post-transplantation (20).

Other investigators showed that between 4 and 18 months after BMT the response to Hib vaccination did not correlate with GVHD, use of immunosuppressive drugs or time to vaccination (25).

The results of these studies indicate that at least two doses of Hib-conjugated vaccine can be administered safely and effectively as early as 4 months after BMT. The impact of donor immunization before marrow harvesting on the appearance of protective antibody levels soon after BMT must be confirmed in larger, prospective, randomized trials before being widely recommended.

\section{Poliovirus vaccine}

Immunity to polio is progressively lost after BMT. Immunocompromised patients and their household contacts should not receive live-attenuated oral poliovirus vaccine. Thus, inactivated poliovirus vaccine (IPV) is recommended after transplantation.

Ljungman et al. (4) demonstrated that $50 \%$ of the patients lost immunity to all three poliovirus types one year after BMT. Patients who received three IPV doses 12, 13 and 14 months after BMT had significantly higher specific antibody titers one year later in comparison to patients who received only one dose. GVHD did not interfere with the response to vaccination when the three-dose regimen was adopted. Other investigators have observed similar findings (26).

More recently, Parkkali et al. (27) compared the response to poliovirus vaccination in 45 patients randomized to receive IPV at 6, 8 and 14 months (early group) after BMT or at 18, 20 and 26 months (late group). Both schedules were similarly immunogenic. Acute GVHD accelerated the decrease of poliovirus antibody titers before vaccination but did not interfere with the response to IPV. Chronic GVHD did not influence the duration of polio immunity or the response to vaccination. These data suggest that poliovirus immunization also does not need to be postponed for more than 6 months after BMT.

\section{Measles vaccine}

Immunity to measles decreases continu- 
ously after BMT $(2,28)$. Although severe measles is expected to occur in immunocompromised patients, there are only two reports in the literature of measles following transplantation $(5,29)$.

Probabilities of measles immunity of about 47, 27 and 20\% have been reported 3, 5 and 7 years after BMT, respectively (30). Among non-vaccinated BMT recipients, Machado et al. (5) observed that $36.6 \%$ were susceptible to measles between the first and second year after BMT and this rate increased to $57.7 \%$ after the second year. Type of BMT (allo or auto), acute or chronic GVHD and the use of immunosuppressive drugs did not influence the persistence of immunity in that series.

The live-attenuated trivalent measlesmumps-rubella vaccine has been administered safely and effectively after the second year of transplantation. Its use has been recommended only in patients not receiving immunosuppressive drugs $(2,7,9,10)$. However, the duration of measles immunity after vaccination and the need for booster doses deserve further investigation in this population.

Among vaccinated patients, Machado et al. (5) observed that $70 \%$ had lost measles immunity 3 years after vaccination, suggesting that serological surveillance to check for immunity should be performed in long-term survivors (Table 2). Moreover, the value and the frequency of booster doses of the vaccine should be better investigated in patients who lost measles immunity.

It is important to stress that although most of the recommendations for BMT recipient vaccination are independent of where in the world the patient lives, there are local variations in the scenario of infections that must be taken into account and adjustments in official guidelines are strongly recommended (31). For example, in 1997, hundreds of BMT recipients were exposed to measles when more than 20,000 cases of measles were diagnosed during an outbreak in the city of São Paulo, Brazil. Eight patients acquired measles and early measles vaccination was the strategy used to avoid the appearance of new measles cases among the patients who had lost specific immunity (5).

To evaluate the safety and effectiveness of this strategy, live-attenuated measlesmumps-rubella vaccine was administered one year after BMT to all patients, even those receiving immunosuppressive drugs. No moderate or severe side effect was noted and all susceptible patients responded to vaccination. The probability of sustained immunity was $60.2 \%$ two years after early vaccination (32). Thus, this strategy can be safely used during outbreaks in countries that have not achieved measles elimination.

\section{Influenza vaccine}

Few data are available concerning influenza vaccination after bone marrow transplantation. Engelhard et al. (33) vaccinated 48 patients with two doses of influenza vaccine administered 2 to 82 months after BMT. Vaccination before the sixth month was totally ineffective and the second dose did not add substantial benefit in terms of specific response and its indication is therefore questionable.

Preliminary results of a study evaluating the use of granulocyte-macrophage colonystimulating factor $(2.5 \mu \mathrm{g} / \mathrm{kg})$ as an immunomodulating factor to enhance the response to

\begin{tabular}{|c|c|c|c|}
\hline \multirow{2}{*}{$\begin{array}{l}\text { Measles } \\
\text { immunity }\end{array}$} & \multicolumn{2}{|c|}{ Years after vaccination (\%) } & \multirow[t]{2}{*}{ Total } \\
\hline & $<3$ years & $\geq 3$ years & \\
\hline Susceptible & $6(27.3)$ & $7(70)$ & 13 \\
\hline Immune & $16(72.7)$ & $3(30)$ & 19 \\
\hline Total & $22(100)$ & $10(100)$ & 32 \\
\hline
\end{tabular}


influenza vaccination showed a limited benefit, mostly in those vaccinated before the first year after BMT. Since side effects were not negligible, its use deserves further investigation (34).

\section{Other vaccines and perspectives}

\section{Varicella vaccine}

No data are available concerning the safety and effectiveness of live-attenuated varicella vaccine before the first year of transplantation, when the risk of varicellazoster virus $(\mathrm{VZV})$ reactivation is higher. Sauerberi et al. (35) did not observe any case of chickenpox or herpes zoster for up to 2 years after vaccination in 15 patients who received one dose of VZV vaccine 12 to 23 months after BMT. These data are difficult to interpret since the occurrence of zoster is expected around the sixth month after transplantation and the risk of a second episode is less than $5 \%$ in this population. Thus, few patients would be really "at risk" after the first year of transplantation and the benefit of late vaccination would be minimal.

Redman et al. (36) heat-inactivated the live-attenuated vaccine and observed diminished clinical severity of zoster in patients who received three doses of the inactivated vaccine 1, 2 and 3 months after BMT. These data suggested that the process of inactivation did not eliminate the immunogenicity of the vaccine, which apparently conferred some protection. Based on this observation, Hata et al. (37) recently demonstrated that four doses of the inactivated varicella vaccine given before hematopoietic cell transplantation and during the first 90 days thereafter (1 dose at $+30,+60$ and +90 ) reduced the risk of zoster in autologous BMT recipients. The protection was correlated with the reconstitution of CD4 T cell immunity against VZV.

The safety and effectiveness of the attenuated varicella vaccine administered to recipients during the first 6 months after autologous and allogeneic BMT remain to be demonstrated in controlled trials.

\section{Hepatitis B and A vaccines}

Other licensed vaccines such as those against hepatitis B and hepatitis A have been recommended on an individual basis.

Hepatitis B vaccine has been recommended after the first year of BMT in countries where the infection is common and children are routinely immunized against hepatitis B.

In the setting of BMT, hepatitis B vaccine has been evaluated in different circumstances: to immunize susceptible patients after transplantation and also to adoptively transfer hepatitis B immunity through vaccination of the donors before marrow harvesting. Surprisingly, few data are available concerning the effectiveness of hepatitis B vaccine in BMT recipients and the duration of immunity after vaccination.

Nagler et al. (38) observed seroconversion rates of about $70 \%$ within 40 days of transplantation in autologous BMT recipients receiving a single dose of hepatitis $B$ vaccine immediately before or after transplantation. Transient seroconversion was seen in about $35 \%$ of the patients.

Ilan et al. (39) demonstrated transfer of hepatitis B immunity in the first 45 days of transplantation from donors vaccinated before marrow harvesting.

Adopting the classical vaccination schedule proposed for immunocompetent hosts, Machado et al. (40) observed 100\% seroconversion in 50 patients vaccinated after the first year of BMT. However, one year after vaccination nearly $60 \%$ of the patients had lost hepatitis B immunity. Sustained immunity was more likely to occur in children and in subjects without chronic GVHD. Interestingly, time to vaccination did not influence the response to vaccination or the duration of immunity, suggesting that no benefit is added by postponing hepatitis $B$ vaccination 
after the first year of BMT.

There are no data on the use of inactivated hepatitis A vaccine in transplant recipients. Considering the efficacy of the vaccine in healthy subjects and the recommendation of vaccination to travelers to endemic areas (41), studies evaluating the safety and effectiveness of hepatitis A vaccine would be of great importance in this population. Children submitted to BMT and recipients traveling to such areas would benefit from hepatitis A vaccination.

\section{References}

1. Lum LG (1987). The kinetics of immune reconstitution after human marrow transplantation. Blood, 69: 369-380.

2. Ljungman $P$, Fridell $E$, Lonnqvist $B$, Bolme $P$, Bottiger $M$, Gahrton $G$, Linde A, Ringden O \& Wahren B (1989). Efficacy and safety of vaccination of marrow transplant recipients with a live attenuated measles, mumps, and rubella vaccine. Journal of Infectious Diseases, 159: 610-615.

3. Ljungman $P$, Wiklund-Hammarsten M, Duraj V, Hammarstrom L, Lonnqvist B, Paulin T, Ringden O, Pepe MS \& Gahrton G (1990). Response to tetanus toxoid immunization after allogeneic bone marrow transplantation. Journal of Infectious Diseases, 162: 496500 .

4. Ljungman P, Duraj V \& Magnius L (1991). Response to immunization against polio after allogeneic marrow transplantation. Bone Marrow Transplantation, 7: 89-93.

5. Machado CM, Gonçalves FB, Pannuti CS, Dulley FL \& Souza VAUF (2002). Measles in bone marrow transplant recipients during an outbreak in São Paulo, Brazil. Blood, 99: 83-87.

6. Ljungman $P$, Cordonnier $C$, De Bock R, Einsele H, Engelhard D, Grundy J, Link H, Locasciulli A, Prentice G \& Reusser P (1995). Immunizations after bone marrow transplantation: results of the European Group for Blood and Marrow Transplantation. Bone Marrow Transplantation, 15: 455-460.

7. Somani J \& Larson RA (1995). Reimmunization after allogeneic bone marrow transplantation. American Journal of Medicine, 98: 389-398.

8. Henning KJ, Whilte MH, Sepkowitz KA \& Armstrong D (1997). A national survey of immunization practices following allogeneic bone marrow transplantation. Journal of the American Medical Association, 277: 1148-1151.

9. Singhal S \& Mehta J (1999). Reimmunization after blood or marrow stem cell transplantation. Bone Marrow Transplantation, 23: 637646.

10. Dykewicz CA, Jaffe HW \& Kaplan JE (2000). Guidelines for preventing opportunistic infections among hematopoietic stem cell transplant recipients. Morbidity and Mortality Weekly Report, 49 (RR-10): $1-128$.

11. Lum LG, Seigneuret MC \& Storb R (1986). The transfer of antigenspecific humoral immunity from marrow donors to marrow recipients. Journal of Clinical Immunology, 6: 389-396.

12. Parkkali T, Ruutu T, Stenvik M, Kuronen T, Kayhty H, Hovi T, Olander RM, Volin L \& Ruutu P (1996). Loss of protective immunity to polio, diphtheria and Haemophilus influenzae type $b$ after allogeneic bone marrow transplantation. Acta Pathologica, Microbiologica et Immunologica Scandinavica, 104: 383-388.

13. Li Volti S, Mauro L, Di Gregorio F, Romeo MA, Lupo L, Pizzarelli G, Mangiagli A, Giammanco G \& Russo G (1994). Immune status and immune response to diphtheria-tetanus and polio vaccines in allogeneic bone marrow-transplanted thalassemic patients. Bone Marrow Transplantation, 14: 225-227.

14. Parkkali T, Olander RM, Ruutu T, Vuontela K, Volin L, Eskola J \& Ruutu P (1997). A randomized comparison between early and late vaccination with tetanus toxoid vaccine after allogeneic BMT. Bone Marrow Transplantation, 19: 933-938.

15. Winston DJ, Ho WG, Schiffman G, Champlin RE, Feig SA \& Gale RP (1983). Pneumococcal vaccination of recipients of bone marrow transplants. Archives of Internal Medicine, 143: 1735-1737.

16. Lortan JE, Vellodi J, Jurges ES \& Hugh-Jones K (1992). Class-and subclass-specific pneumococcal antibody levels and response to immunization after bone marrow transplantation. Clinical and Experimental Immunology, 88: 512-519.

17. Hammarstrom V, Pauksen K, Azinge J, Oberg G \& Ljungman $P$ (1993). Pneumococcal immunity and response to immunization with pneumococcal vaccine in bone marrow transplant patients: the influence of graft versus host reaction. Supportive Care in Cancer, 1: 195-199.

18. Parkkali T, Kayhty H, Ruutu T, Volin L, Eskola J \& Ruutu P (1996). A comparison of early and late vaccination with Haemophilus influenzae type $\mathrm{b}$ conjugate and pneumococcal polysaccharide vaccines after allogeneic BMT. Bone Marrow Transplantation, 18: 961-967.

19. Spoulou V, Victoratos $P$, loannidis JPA \& Grafakos S (2000). Kinetics of antibody concentration and avidity for the assessment of immune response to pneumococcal vaccine among children with bone marrow transplants. Journal of Infectious Diseases, 182: 965969.

20. Molrine DC, Guinan EC, Antin JH et al. (1996). Donor immunization with Haemophilus influenzae type b (HIB)-conjugate vaccine in allogeneic bone marrow transplantation. Blood, 87: 3012-3018.

21. Avanzini MA, Carra AM, Maccario R, Zecca M, Pignatti $P$, Marconi M, Comoli P, Bonetti F, De Stefano P \& Locatelli F (1995). Antibody response to pneumococcal vaccine in children receiving bone marrow transplantation. Journal of Clinical Immunology, 15: 137-144.

22. Molrine DC, Antin JH, Guinan EC, Soiffer RJ, MacDonald K, Malley R, Malinoski F, Trocciola S, Wilson M \& Ambrosino DM (2003). Donor immunization with pneumococcal conjugate vaccine and early protective antibody responses following allogeneic hematopoietic cell transplantation. Blood, 101: 831-836.

23. Guinan EC, Molrine DC, Antin JH, Lee MC, Weinstein HJ, Sallan SE, Parsons SK, Wheeler C, Gross W \& McGarigle C (1994). Polysaccharide conjugate vaccine responses in bone marrow transplant patients. Transplantation, 57: 677-684.

24. Vance E, George S, Guinan EC, Wheeler C, Antin JH, Ambrosino DM \& Molrine DC (1998). Comparison of multiple immunization schedules for Haemophilus influenzae type-b conjugate and teta- 
nus toxoid vaccines following bone marrow transplantation. Bone Marrow Transplantation, 22: 735-741.

25. Barra A, Cordonnier C, Preziosi MP, Intrator L, Hessel L, Fritzell B \& Preud'homme JL (1992). Immunogenicity of Haemophilus influenzae type b conjugate vaccine in allogeneic bone marrow recipients. Journal of Infectious Diseases, 166: 1021-1028.

26. Engelhard D, Handsher R, Naparstek E, Hardan I, Strauss N, Aker M, Or R, Baciu H \& Slavin S (1991). Immune response to polio vaccination in bone marrow transplant recipients. Bone Marrow Transplantation, 8: 295-300.

27. Parkkarli T, Stenvik M, Ruutu T, Hovi T, Volin L \& Ruutu P (1997). Randomized comparison of early and late vaccination with inactivated poliovirus vaccine after allogeneic BMT. Bone Marrow Transplantation, 20: 663-668.

28. Pauksen K, Duraj V, Ljungman P, Sjolin J, Oberg G, Lonnerholm G, Fridell E, Smedmyr B \& Simonsson B (1992). Immunity to and immunization against measles, rubella and mumps in patients after autologous bone marrow transplantation. Bone Marrow Transplantation, 9: 427-432

29. Nakano T, Shimono Y, Sugiyama K et al. (1996). Clinical features of measles in immunocompromised children. Acta Paediatrica Japonica, 38: 212-217.

30. Ljungman P, Lewensohn-Fuchs I, Hammarstrom V, Aschan J, Brandt L, Bolme P, Lonnqvist B, Johansson N, Ringden O \& Gahrton G (1994). Long-term immunity to measles, mumps, and rubella after allogeneic bone marrow transplantation. Blood, 84: 657-663.

31. Ljungman P (1999). Immunization of transplant patients. Bone Marrow Transplantation, 23: 635-636.

32. Machado CM, Sumita LM, Rocha IF, Pannuti CS \& Souza VAUF (2002). Early measles vaccination in BMT recipients. The 12th International Symposium on Infections in the Immunocompromised Host. Bergen, Norway, June 23-26, 2002.

33. Engelhard D, Nagler A, Hardan I, Morag A, Aker M, Baciu H, Strauss N, Parag G, Naparstek E \& Ravid Z (1993). Antibody response to a two-dose regimen of influenza vaccine in allogeneic $\mathrm{T}$ cell-depleted and autologous BMT recipients. Bone Marrow Transplantation, 11: 1-5.

34. Pauksen K, Linde A, Hammarstrom V, Sjolin J, Carneskog J, Jonsson G, Oberg G, Engelmann H \& Ljungman P (2000). Granulocytemacrophage colony-stimulating factor as immunomodulating factor together with influenza vaccination in stem cell transplant patients. Clinical Infectious Diseases, 30: 342-348.

35. Sauerberi A, Prager J, Hengst U, Zintl F \& Wutzler P (1997). Varicella vaccination in children after bone marrow transplantation. Bone Marrow Transplantation, 20: 381-383.

36. Redman RL, Nader S, Zerboni L, Liu C, Wong RM, Brown BW \& Arvin AM (1997). Early reconstitution of immunity and decreased severity of herpes zoster in bone marrow transplant recipients immunized with inactivated varicella vaccine. Journal of Infectious Diseases, 176: 578-585.

37. Hata A, Asanuma H, Rinki M, Sharp M, Wong RM, Blume K \& Arvin AM (2002). Use of inactivated varicella vaccine in recipients of hematopoietic-cell transplants. New England Journal of Medicine, 347: 26-34.

38. Nagler A, Ilan Y, Adler R, Or R, Naparstek E, Shouval D \& Slavin S (1995). Successful immunization of autologous bone marrow transplantation recipients against hepatitis B virus by active vaccination. Bone Marrow Transplantation, 15: 475-478.

39. Ilan Y, Nagler A, Adler R, Naparstek E, Or R, Slavin S, Brautbar C \& Shouval D (1993). Adoptive transfer of immunity to hepatitis B virus after T cell-depleted allogeneic bone marrow transplantation. Hepatology, 18: 246-252.

40. Machado CM, Rocha IF, Diomede B et al. (1996). Effectiveness of hepatitis B vaccination and persistence of immunity after BMT (Abstract). The Ninth International Symposium on Infections in the Immunocompromised Host. Assisi, Italy, June 23-26, 1996.

41. Wilson ME (2001). Travel-related vaccines. Infectious Disease Clinics of North America, 15: 231-251. 